\title{
DiAgnOSe THIS
}

\section{A 63-Year-Old Man with Lightheadedness and Flank Pain}

\author{
Adam Dmytriw ${ }^{1}$, Patrick Casey MD², Christopher Lightfoot MD $^{3}$ \\ ${ }^{1}$ Class of 2014, Faculty of Medicine, Dalhousie University \\ ${ }^{2}$ Division of Vascular Surgery, Department of Surgery, Dalhousie University \\ ${ }^{3}$ Department of Radiology, Dalhousie University
}

A 63-year-old man presents to the emergency department with a 3-hour history of sudden-onset sharp left flank pain radiating to the left lower quadrant. This is accompanied with nausea and vomiting. He also complains of lightheadedness as well as general weakness over the same time period. He has had documented hypertension for the past 10 years and has a 60 pack-year smoking history.

$\mathrm{He}$ is pale and diaphoretic. His abdomen is soft, non-tender, with no palpable abnormalities. His physical examination is somewhat limited by his obese body habitus. His peripheral pulses are diminished. His vital signs are: HR 72, RR 16, BP 78/60, $\mathrm{O}_{2}$ sat 99\%. An enhanced computed tomography (CT) scan was obtained (Figure 1).

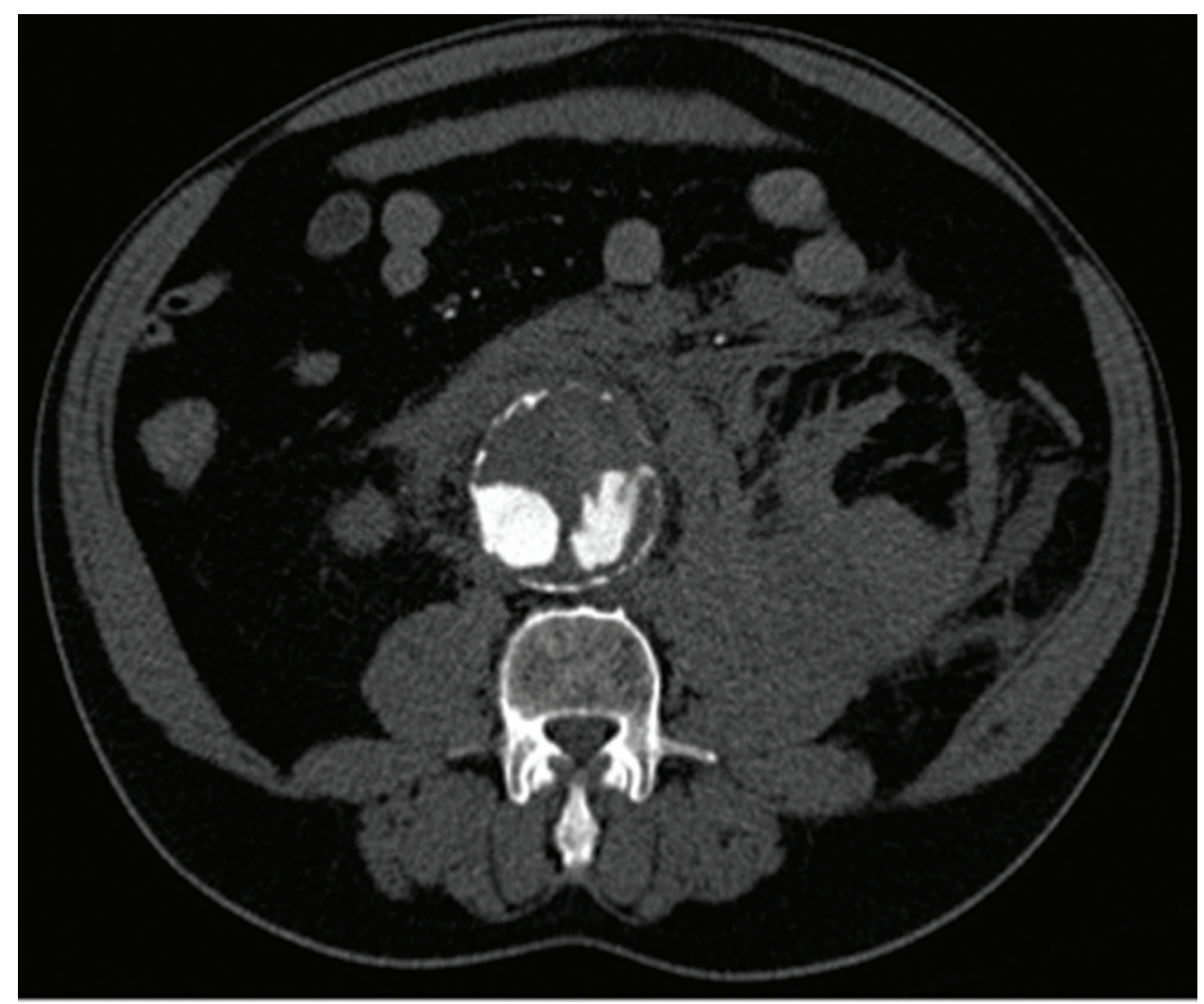

Figure 1. Axial image from a contrast-enhanced abdominal CT performed at admission. 


\section{Choose the BEST answer:}

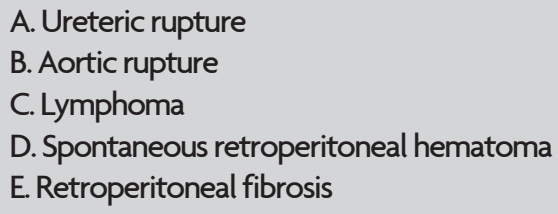

Diagnosis:

Aortic rupture

\section{Overview}

Abdominal aortic aneurysm (AAA) occurs as a result of aortic wall degeneration. An aneurysm is typically described as an increase in diameter greater than $50 \%$ of the normal vessel calibre. Of the $40 \%$ of individuals who survive aneurysm rupture long enough to seek medical attention, 50-60\% will survive operative repair. ${ }^{1-4}$ The incidence of AAA sharply climbs in individuals from the sixth decade of life onwards. Guidelines now suggest a one-time screening of men between the ages of $65-75 .{ }^{5}$

\section{Imaging Findings}

The majority of AAAs are found incidentally during medical imaging and routine physical exams for various causes but many are now found with focussed screening. Ultrasound is the screening modality of choice and is often readily available to assess patients on an emergency basis. It can be invaluable to confirm the presence of an abdominal aneurysm in an unstable patient with a suspected rupture. It is limited in evaluating the size and morphology of aneurysms as it can be challenging to see mural thrombus and wall thickness of vessels. CT is very often used in stable patients as it is excellent at characterizing aneurysms and planning treatment. It accurately depicts the size and extent of the aneurysm and allows assessment of the feasibility of endovascular versus open repair techniques. ${ }^{6}$

CT imaging findings of AAA rupture include active contrast extravasation, retroperitoneal hematoma (high density fluid) and aortic mural irregularities. Fistulous communications can also be seen. A rapidly expanding aneurysm and discontinuity of mural calcification are signs of instability on CT. Signs of impending rupture include the "draped aorta" sign (aortic wall conforms to the adjacent vertebral body), high attenuation in the aortic wall on unenhanced $\mathrm{CT}$, and focal pointing of the wall. ${ }^{7}$

In this above case, there is an abdominal aneurysm with prominent mural calcification. The dense fluid seen in the retroperitoneal spaces and along the fascial planes, is compatible with hematoma. The high density luminal contrast points to the aortic wall in a region of focal mural calcification discontinuity (Figure 2).

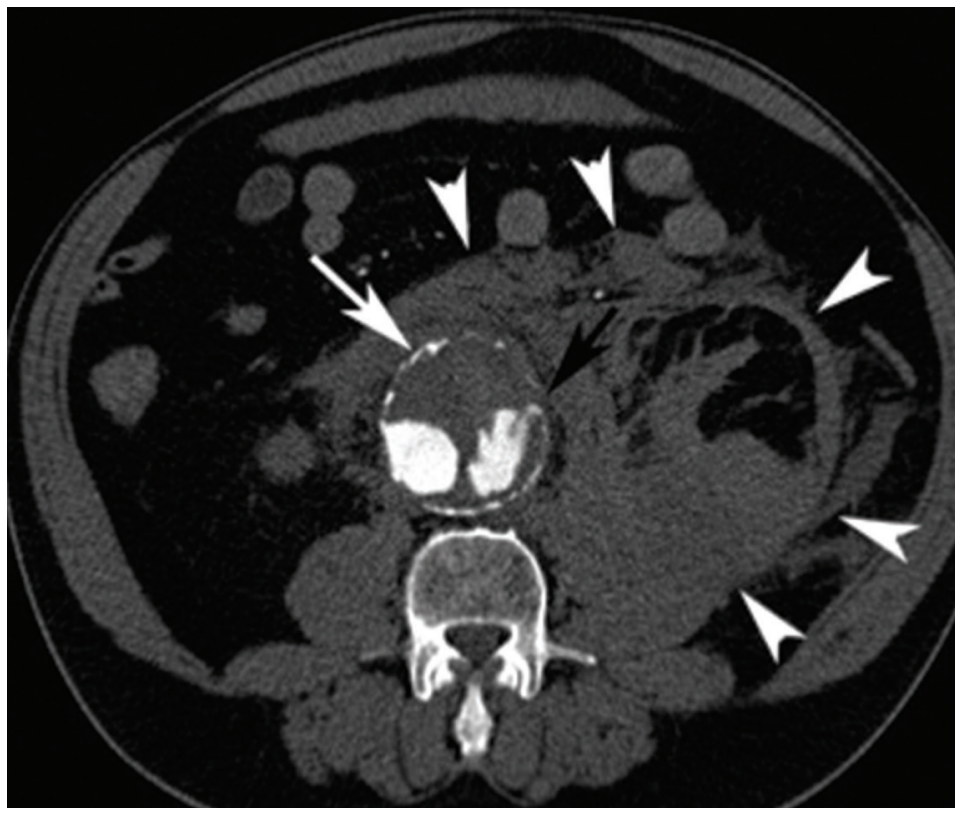

Figure 2. The same abdominal CT image. There is an abdominal aneurysm with prominent mural calcification (white arrow). The dense fluid seen in the retroperitoneal spaces and along the fascial planes, is compatible with hematoma (white arrowheads). The high density luminal contrast points to the aortic wall in a region of focal mural calcification discontinuity (black arrow). 


\section{Clinical Features}

The most common presenting symptom for an abdominal aortic aneurysm is abdominal pain, which may radiate to the flank, back or groin. At the point of rupture, a classical triad of hypotension, pain, and a pulsatile abdominal mass is noted in as many as one-third of patients. ${ }^{3}$

\section{Management}

Although there is little survival advantage in treating elective AAAs smaller than $5.5 \mathrm{~cm}$, all cases of ruptured aneurysms require treatment. Both open and endovascular techniques for repair are acceptable and are regularly employed, with endovascular aneurysm repair (EVAR) beginning to demonstrate lower rates of perioperative morbidity and mortality as more data becomes available. Endovascular repair in selected patients may be associated with a trend towards reductions in blood loss, duration of intensive care treatment, and mortality. ${ }^{1}$

Initial management steps include immediately contacting vascular surgery and active resuscitation with fluids and blood products to maintain blood pressure between 80 to $100 \mathrm{mmHg}$.

\section{References}

1. Dillon M, Cardwell C, Blair PH, Ellis P, Kee F, Harkin DW. Endovascular treatment for ruptured abdominal aortic aneurysm. Cochrane Db Syst Rev 2007;1:1-18.

2. Farooq MM, Freischlag JA, Seabrook GR, Moon MR, Aprahmin C, Towne JB. Effect of the duration of symptoms, transport time, and length of emergency room stay on morbidity and mortality in patients with ruptured abdominal aortic aneurysms. Surgery 1996;119: 9-14.

3. Ernst CB. Abdominal Aortic-Aneurysm. New Engl J Med 1993;328: 1167-1172.

4. Bown MJ, Sutton AJ, Bell PRF, Sayers RD. A meta-analysis of 50 years of ruptured abdominal aortic aneurysm repair. Brit J Surg 2002;89:714-730.

5. Hallett JW, Mills JL, Earnshaw J, Reekers JA, Rooke T. Comprehensive Vascular and Endovascular Surgery. 2nd Edition edn 2009.

6. Litmanovich D, Bankier AA, Cantin L, Raptopoulos V, Boiselle PM. CT and MRI in Diseases of the Aorta. Am J Roentgenol 2009;193: 928-940.

7. Souza D, Ledbetter S. Diagnostic Errors in the Evaluation of Nontraumatic Aortic Emergencies. Semin Ultrasound 2012;33: 318-336.
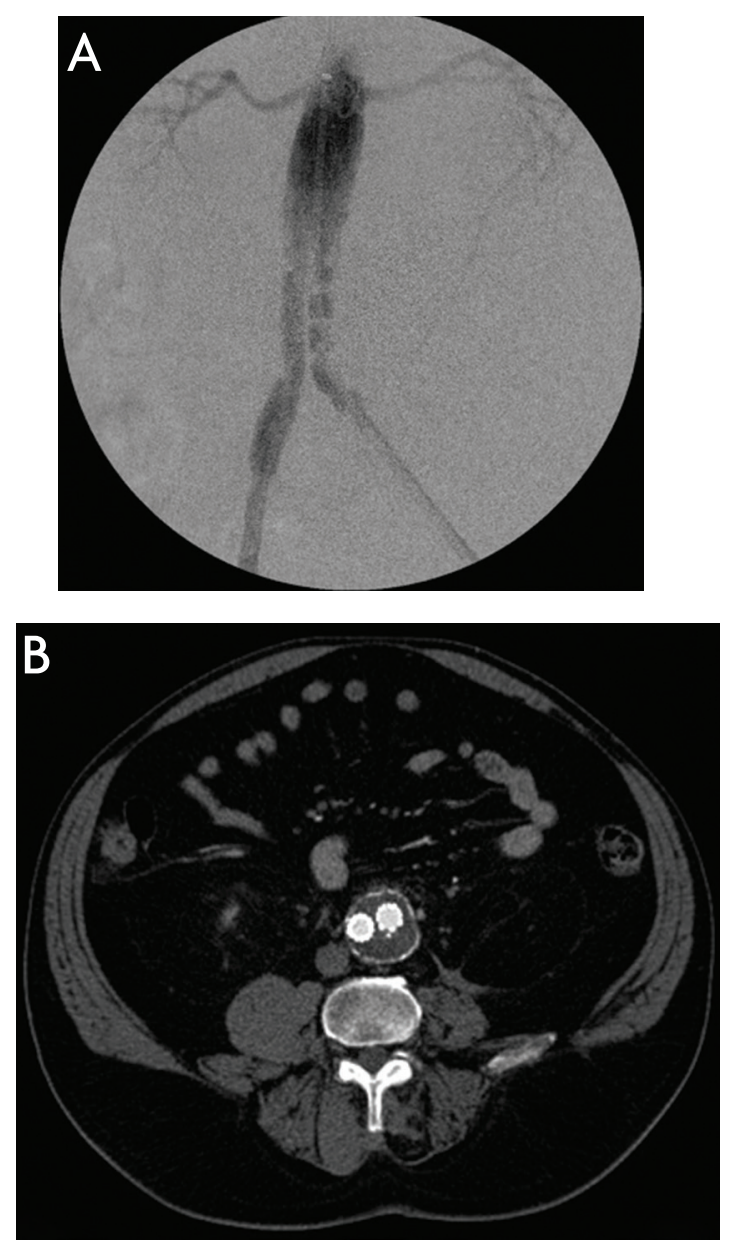

Figure 3.

(A) Fluoroscopic digital subtraction image taken during contrast injection after the deployment of an abdominal aortic endograft. Note that the native aortic aneurysm sac is excluded and contrast is seen only in the main body and limbs of the endograft below the level of the renal arteries.

(B) Axial CT image from an arterial enhanced study two years following the endovascular repair of the aneurysm. Note the resorption of the hematoma in the retroperitoneum. Additionally, the native aortic aneurysm sac (noted by the calcified wall) is much smaller in diameter than on the initial study. 